\title{
CATALOGAÇÃO DE OBJETOS A PARTIR DE IMAGENS GEORREFERENCIADAS OBTIDAS COM UM SISTEMA MÓVEL DE MAPEAMENTO
}

\section{CATALOGING OBJECTS FROM GEORREFERENCED IMAGES OBTAINED WITH A MOBILE MAPPING SYSTEM}

Elivânia Barros de Oliveira ${ }^{1}$; Francisco Assis da Silva ${ }^{1}$; Mário Augusto Pazoti $^{1}$; Leandro Luiz de Almeida ${ }^{1}$; Danillo Roberto Pereira ${ }^{1}$; Ricardo Luiz Barbosa ${ }^{2}$

${ }^{1}$ Universidade do Oeste Paulista - UNOESTE, Faculdade de Informática - FIPP,
Presidente Prudente, SP. ${ }^{2}$ Universidade Estadual Paulista - UNESP, Campus de
Sorocaba, SP. e-mail: $\underline{\text { danilopereira@unoeste.br }}$

RESUMO - O avanço da tecnologia tem contribuído com diversas áreas na cartografia, proporcionando o surgimento dos Sistemas de Informação Geográfica (SIG), os quais são poderosas ferramentas utilizadas para organizar, administrar e processar grandes volumes de dados, que são obtidos por Sistemas Móveis de Mapeamento (SMM). Este trabalho está focado no gerenciamento desses dados e georreferenciamento de objetos desejados encontrados nas vias. Árvores, postes, lixeiras, pontos de ônibus são exemplos de objetos, que podem ser descritos, e com suas coordenadas de posicionamento calculadas podem ser representados em um mapa, sendo de grande valia para questões de localização e mensuração desses objetos. Palavras-chave: Fototriangulação, Georreferenciamento, Sistemas Móveis de Mapeamento, Sistemas de Informação Geográfica.

ABSTRACT - The advances in technology has contributed in many areas in cartography, providing the appearance of Geography Information Systems (GIS's) that are powerful tools to organize, manage and process huge data values obtained by Mapping Mobile Systems (MMS). This work is focused on the management of these data and georeferencing of desired objects found on roads. Trees, lamppost, garbage can, bus stops are examples of objects, that can be described, and their position coordinates can be represented in a map, being of a great value for localization and measuring of these objects.

Keywords: Phototriangulation, Georeferencing, Mobile Mapping Systems, Geographic Information Systems.

Recebido em: 02/10/2014

Revisado em: 30/10/2014

Aprovado em: 25/11/2014 


\section{INTRODUÇÃO}

Um Sistema de Informação Geográfica (SIG ou GIS - Geographic Information System) é um sistema que permite analisar, organizar a representação do espaço e dos fenômenos que nele ocorrem. Uma forma muito utilizada de obtenção de dados para estudo pelos SIG são os Sistemas Móveis de Mapeamento (SMM) (SILVA et al., 2001). Os SMM são sistemas que capturam dados para análise e medições dos pontos para fins de levantamento e mapeamento fotogramétrico ou, simplesmente, para avaliação das vias de transporte. Os SMM são dotados de câmaras fotográficas e de vídeo, GPS, e muitas vezes por uma Unidade de Medida Inercial (IMUInercial Navigation System), responsável por auxiliar o GPS em lugares onde pode ser perdida a localização, por exemplo, em vias que possuem um número muito elevado de prédios ou árvores. Na Figura 1 é mostrado um GPS profissional utilizado em um SMM, já na Figura 2 é mostrada uma IMU.

Segundo Oliveira e Silva (2001) as câmaras são o meio mais eficiente de se obter imagens de um objeto a ser mapeado, as câmaras podem ser embutidas em aeronaves ou mesmo no teto de um veículo. A aerofotografia, por sua vez, é o meio mais eficiente de se obter dados para realização de mapeamentos topográficos.

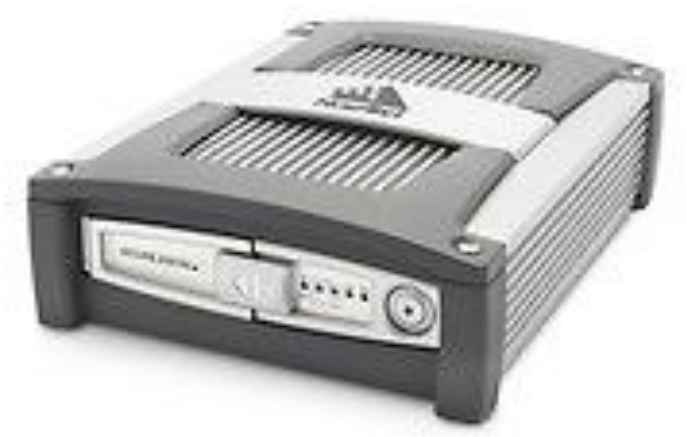

Figura 1. GPS profissional.

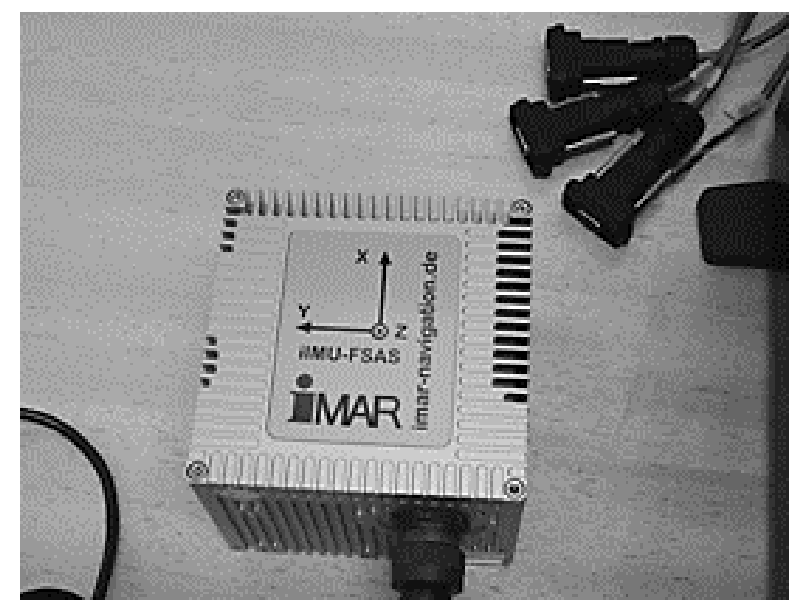

Figura 2. Unidade de Medida Inercial.

Um método que vem sendo muito utilizado para levantamento atualmente são os SMM instalados em veículos automotores terrestres, que podem ser denominados de Mapeamento Fotogramétrico Móvel. Eles correspondem a uma linha de investigação científica e tecnológica de mapeamento de vias terrestres de locomoção (rodovias, ruas, avenidas) que vem sendo muito utilizado para fins de pesquisa e até comercialmente, que também podem ser chamadas de Unidade Móvel de Mapeamento Digital 
(UMMD). Na Figura 3 é mostrado um protótipo de uma UMMD.

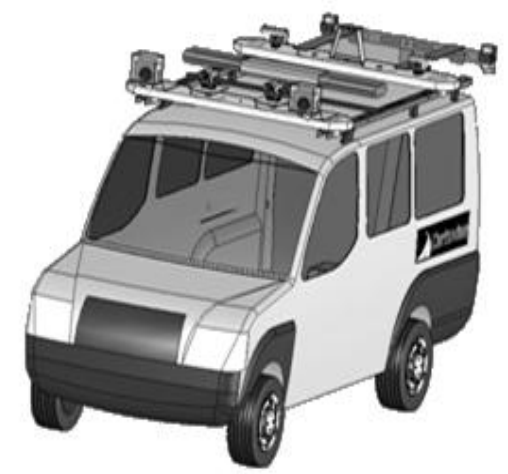

Figura 3. Protótipo de UMMD.

As imagens coletadas por tais equipamentos são chamadas de imagens georreferenciadas. Quando se diz que uma imagem, mapa ou qualquer forma de informação geográfica são georreferenciadas, pode-se dizer que esses objetos têm suas coordenadas conhecidas em um dado sistema de referência. O modelo do par de câmaras utilizadas no levantamento deste trabalho foram NIKON D300S (Figura 4). Essas câmaras possuem 12 Megapixels de resolução geométrica, posicionadas no teto do veículo com o eixo óptico paralelo ao deslocamento.

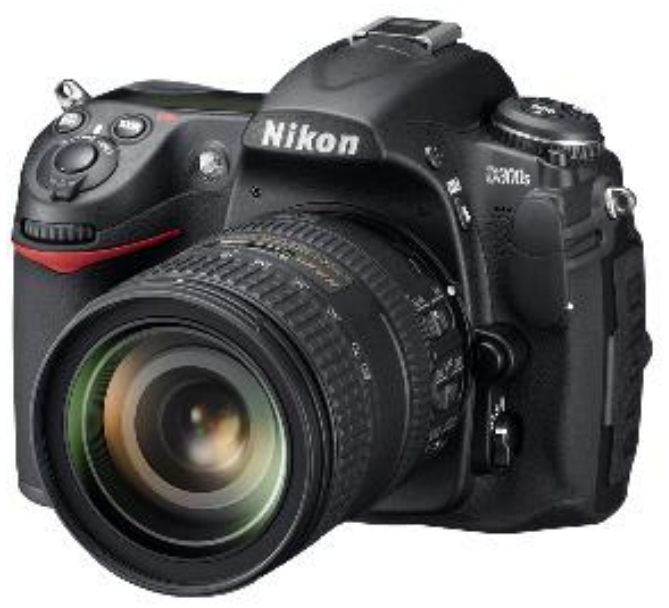

Figura 4. NIKON D300S.

O objetivo deste trabalho está focado na construção de um sistema de gerenciamento de imagens e dados providos por meio de levantamentos cartográficos e também resultantes de aplicações que tratam esses dados. Um exemplo desse tipo de levantamento é a marcação das posições georreferenciadas de árvores, postes, placas de sinalização, pontos de ônibus, lixeiras e muitos outros objetos em uma base cartográfica. Para as posições dos objetos no mapa foram usadas as latitudes, longitudes e altitudes dos objetos a partir dos pares de imagens fornecidas por meio de levantamento utilizando um SMM.

A partir do sistema proposto, dados e imagens, como no exemplo apresentado, podem ser buscados, consultados e visualizados em um mapa com suas posições reais, podendo, assim, ter um levantamento de algum objeto que se deseja em uma cidade. 
Este processo se inicia com a obtenção das coordenadas, pontos na imagem ou mapa, conhecidos como pontos homólogos. Após a obtenção dos pontos na imagem, utilizam-se algoritmos de busca para o cálculo das coordenadas dos pontos marcados no mundo real utilizando a latitude e longitude do objeto para se ter uma camada em um mapa com a visualização destes objetos.

Este trabalho está organizado da seguinte forma: Na Seção 2 é apresentado um aspecto global sobre o Sistema de Posicionamento Global (GPS) como são organizados os satélites na constelação e como o GPS foi desenvolvido pelo Departamento de Defesa dos Estados Unidos, essa seção apresenta ainda temas relacionados à latitude, longitude, altitude, geoide e aborda também uma descrição das Unidades de Medida Inerciais, como elas ajudam o GPS a se localizar quando o mesmo perde a localização; A Seção 3 aborda os recursos que foram utilizados para gerar a camada do mapa de visualização dos objetos que foram descritos, e como o Google Maps disponibiliza informações para os desenvolvedores utilizarem os recursos de mapas em aplicações; A Seção 4 aborda a idealização da aplicação, sobre como surgiu a ideia e uma breve informação de como funciona a aplicação; A Seção 5 trata dos experimentos que foram realizados com a ferramenta; Na Seção 6 é apresentada uma breve amostra dos resultados obtidos com a utilização da aplicação e a visualização do mapa resultante da aplicação com os objetos descritos; A Seção 7 apresenta a conclusão da aplicação, e também apresenta os aspectos que ficaram em aberto como sugestões para o direcionamento de trabalhos futuros.

\section{SISTEMA DE POSICIONAMENTO GLOBAL (GPS)}

O Sistema de Posicionamento Global é um sistema de rádio-navegação que também pode ser denominado NAVSTAR-GPS (Navigation Satellite with Time and Raging), desenvolvido pelo Departamento de Defesa dos Estados Unidos com a finalidade de navegação, posicionamento topográfico entre outros (MONICO, 2000). É alimentado por um sistema de 24 satélites distribuídos em seis planos orbitais igualmente espaçados (Quatro satélites em cada plano) numa altitude com aproximação de $20.200 \mathrm{Km}$. Os planos orbitais têm uma inclinação de $55^{\circ}$ com relação ao equador e o período orbital é de aproximadamente 12 horas siderais. $\mathrm{Na}$ Figura 5 é demonstrada a constelação de satélites utilizada pelo GPS. 


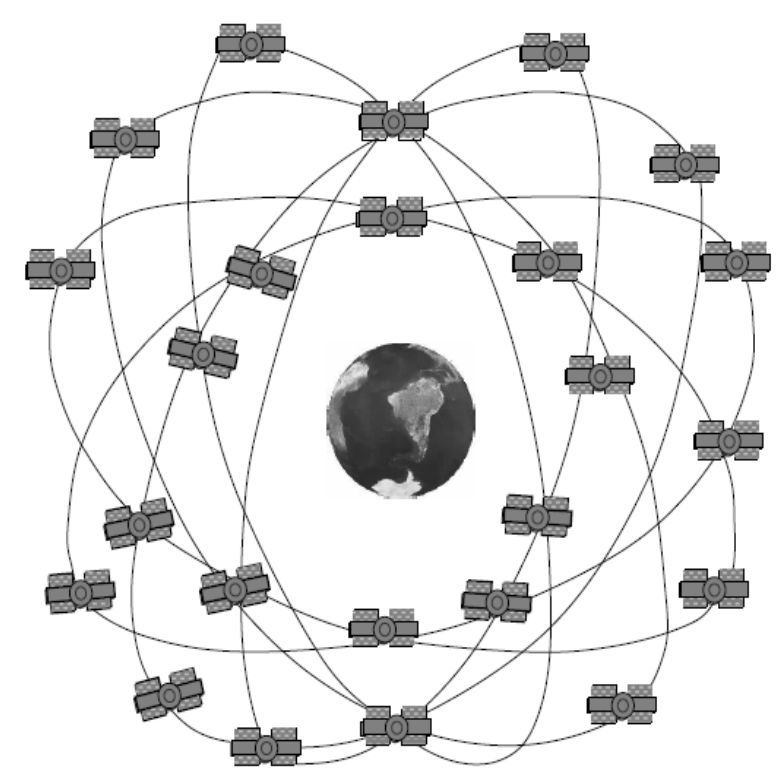

Figura 5. Constelação de Satélites.

Fonte: (MONICO, 2000).

\subsection{Latitude}

A Latitude é a distância em relação à linha do Equador medida ao longo do meridiano de Greenwich. Essa distância é medida em graus, que pode variar entre $0^{\circ}$ a $90^{\circ}$ para o Norte ou para o Sul. Por exemplo, Lisboa está à latitude de $38^{\circ} 4 N$. O Rio de Janeiro a latitude de $22^{\circ} 55 \mathrm{~S}$ e Macau a latitude de $22^{\circ} 27$ N (CIÊNCIA... 2013).

$\mathrm{Na}$ Figura 6 é mostrado como funciona a latitude, indicando por $N$ os graus ao Norte e $S$ os graus ao Sul tendo como referência a linha do Equador.

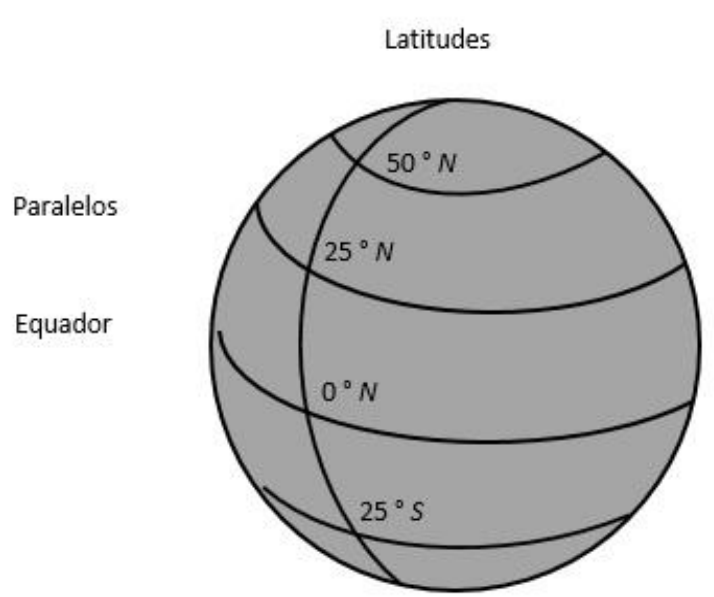

Figura 6. Latitude.

Fonte: (Ciência Viva, 2013).

\subsection{Longitude}

A longitude é a distância em relação ao Meridiano de Greenwich medida ao longo da linha do Equador. Essa distância é dada em graus, podendo variar entre $0^{\circ}$ e $180^{\circ}$ para Leste ou para Oeste. Lisboa está a $9^{\circ} 8$ $W$, o Rio de Janeiro a longitude $34^{\circ} 53 \mathrm{~W}$ e Macau a longitude $113^{\circ} 53$ E. Na Figura 7 é mostrada a representação da longitude, indicando por $W$ os Graus para Oeste e por $E$ os graus para Leste (CIÊNCIA... 2013).

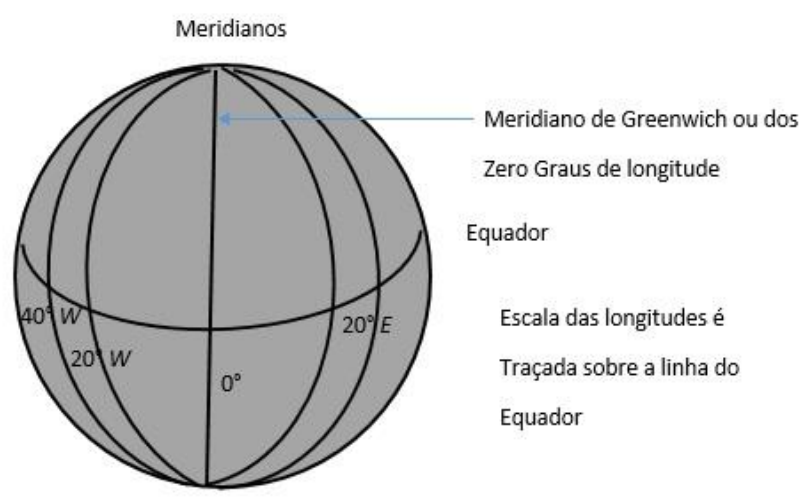

Figura 7. Longitude.

Fonte: (Ciência Viva, 2013). 


\subsection{Geoide}

Etimologicamente significa forma da Terra que é definida pela linha do prolongamento do nível médio dos mares e continentes. É a representação mais próxima da realidade física expressa pelo campo gravitacional terrestre. Sendo uma superfície definida a partir do campo de gravidade, o geoide tem intrínseca relação com a determinação de altitudes (ROQUE et al., 2006).

\subsection{Altitude}

A Terra é aproximadamente esférica, com um ligeiro achatamento no geoide. Para que seja obtida a altitude de um ponto sobre a terra define-se um geoide, com um raio de $6378 \mathrm{~km}$. A altitude de um ponto na Terra é a distância na vertical à altitude desse geoide. A altitude média do aeroporto de Lisboa é de 114 m, mas a altitude média da Holanda é negativa (CIÊNCIA... 2013).

\subsection{Unidade de medida inercial}

O sistema de medida inercial é composto por uma unidade de medida inercial (IMU-Inercial Measurement Unit) e um processador de navegação. A unidade é composta por três acelerômetros que medem acelerações lineares com respeito a um referencial inercial, três giroscópios que medem razões angulares percebidas pela IMU, além de sensores auxiliares, para a calibração. Os principais sensores, responsáveis pela calibração da altitude são os giroscópios, que medem as inclinações da plataforma ao longo do tempo (RUY, 2004).

$$
\text { Segundo Silva (2012) os sensores }
$$
inerciais (IMU) têm basicamente duas funções em determinação da altitude (ângulos de orientação) do sensor de imagem (câmaras) e prover a posição quando o sinal do GPS é perdido por algum motivo, principalmente por obstrução de edificações, árvores, túneis etc. A primeira mede a rotação do sistema referencial da imagem em relação ao referencial fixo e a outra mantém a posição do sensor conhecida. De uma maneira simples, o sistema de medida inercial é um conjunto de instrumentos projetados para registrar as variações do estado de inércia de um corpo rígido, a fim de controlar ou manipular sua trajetória.

Segundo Cramer e Stallmann (2001) o Sistema Inercial pode complementar o GPS fornecendo as informações de posição inicial e de velocidade após a perda de sinal do receptor, mesmo quando a visibilidade dos satélites é insuficiente (menos de quatro satélites visíveis). O sistema de sensores inerciais pode ser usado para interpolar as posições do GPS quando a recepção de um dado satélite for obstruída. Por outro lado, o bom desempenho do sistema GPS pode suplementar o sistema de navegação inercial por meio da estimação acurada do comportamento de seus erros ao longo do 
tempo. Estes erros estão relacionados ao problema de deriva das informações de trajetória inerente aos sistemas inerciais. Os erros internos do sistema inercial são modelados como desvios nos giros e deslocamentos nos acelerômetros e, são corrigidos com a filtragem de Kalman (KALMAN, 1960), a partir das informações provenientes de posições do GPS.

\section{RECURSOS E MAPAS}

Para tornar possível a obtenção dos pontos descritos em um mapa representando a posição de determinado objeto no mundo real foi utilizado o serviço Google Maps, para se criar aplicativos com base em localização. Para isso foi utilizada uma API (Application Programming Interface) denominada Google Places. Segundo o Guia... (2013) a API do Google Places é um serviço que retorna informações sobre locais (definidos como estabelecimentos, localizações geográficas ou pontos de interesse conhecidos) utilizando solicitações HTTP. Essas solicitações de locais são realizadas com base nas coordenadas de latitude/longitude, sendo o formato da URL para essa requisição apresentado a seguir.

https://maps.googleapis.com/maps/a pi/place/details/output?parameters

Nessa URL output pode ter um dos seguintes valores:
"Json", que indica saída em JSON (JavaScript Object Notation) ou "Xml", que indica saída como XML.

Existem alguns parâmetros necessários para iniciar uma solicitação de pesquisa. As URLs têm como padrão todos os parâmetros separados pelo caractere ' $\&$ '. A lista de parâmetros e seus valores possíveis são apresentadas a seguir:

Key (obrigatório): A chave de API do aplicativo. Essa chave tem por objetivo identificar o aplicativo para fins de gerenciamento de cota e para que locais adicionados a partir do aplicativo sejam disponibilizados imediatamente a ele.

Reference (obrigatório): identificador textual exclusivo para um local obtido, a partir de uma solicitação de pesquisa local.

Sensor (obrigatório): indica se a solicitação de detalhes do lugar veio ou não de um dispositivo que utiliza um sensor de localização (como um GPS). Esse valor deve receber os valores true ou false.

Language (opcional): o código do idioma, indicando em qual idioma os resultados devem ser retornados.

O exemplo a seguir é um exemplo de solicitação:

https://maps.googleapis.com/ maps/api/place/details/json?referenc $e=C m R Y A A A A$ ciqGsTRX1mXRvuXSH2Er WWjCINE1aLIWP64MCWDN5vkXvXoQ GPKIdMfmdGyqWSpm7BEYCgDmiv7K 
c2PF7QA7brMAwBbAcqMr5i1f4PwTp aovIZjysCEZTry8Ez30wpEhCNCXpynext Cld2EBsDkRKsGhSLayuRyFsex6JA6NPh 9dyupoTH3g\&sensor=true \&key=Add $Y$ ourOwnKeyHere

Uma resposta JSON contém os seguintes elementos, chamados elementos raiz:

Status: Contém metadados na solicitação.

Result: contém informações detalhadas sobre a solicitação realizada.

Html_attributions: contém um conjunto de atribuições sobre essa listagem a ser exibido para o usuário.

Uma resposta XML consiste em um único parâmetro (PlaceDetaisResponse) e em três elementos de nível superior:

Status: também contém metadados na solicitação.

Result: elemento que contém informações detalhadas sobre apenas um estabelecimento.

Html_attributions um conjunto de atribuições a ser exibido para o usuário.

Códigos de Status: contém o status da solicitação e pode conter informações de depuração como, por exemplo, o motivo da solicitação de local ter falhado. O campo status pode ter os valores a seguir:

$O K$ : indica que o local foi detectado e pelo menos um resultado foi retornado sem erro.
UNKNOWN_ERROR: indica um erro no servidor, nesse caso deve-se tentar novamente.

ZERO_RESULTS: indica que a referência era válida, mas não se refere a um resultado válido.

OVER_QUERY_LIMIT: indica que houve uma ultrapassagem de cota.

REQUEST_DENIED: indica que a solicitação foi negada, pode indicar a falta de um parâmetro sensor.

INVALID_REQUEST: indica que a consulta está ausente.

NOT_FOUND: indica que o local, não foi encontrado na base de dados do Google Places.

Depois de marcados os pontos desejados dos objetos nas imagens georreferenciadas, a aplicação realiza os cálculos das coordenadas do objeto selecionado para permitir que sejam visualizados em uma camada de um mapa. Para isso uma solicitação HTTP é enviada para a base de dados do Google Places e a mesma é devolvida para aplicação com os pontos dos objetos no local com suas coordenadas exatas.

Os serviços Google Static Maps API, Google Geocoding API e Google Directions API, do recurso Google Maps API, é um recurso gratuito, para qualquer site ou aplicação. Na Figura 8 é demonstrado o 
esquema de utilização da API do Google Places em uma aplicação desktop.

A aplicação acessa a base de dados para ter acesso a latitude e longitude dos pontos, por meio de requisição SQL, após receber os dados a aplicação envia uma URL para o Google Places, Caso a resposta seja afirmativa o navegador Web abrirá a camada de visualização do mapa.

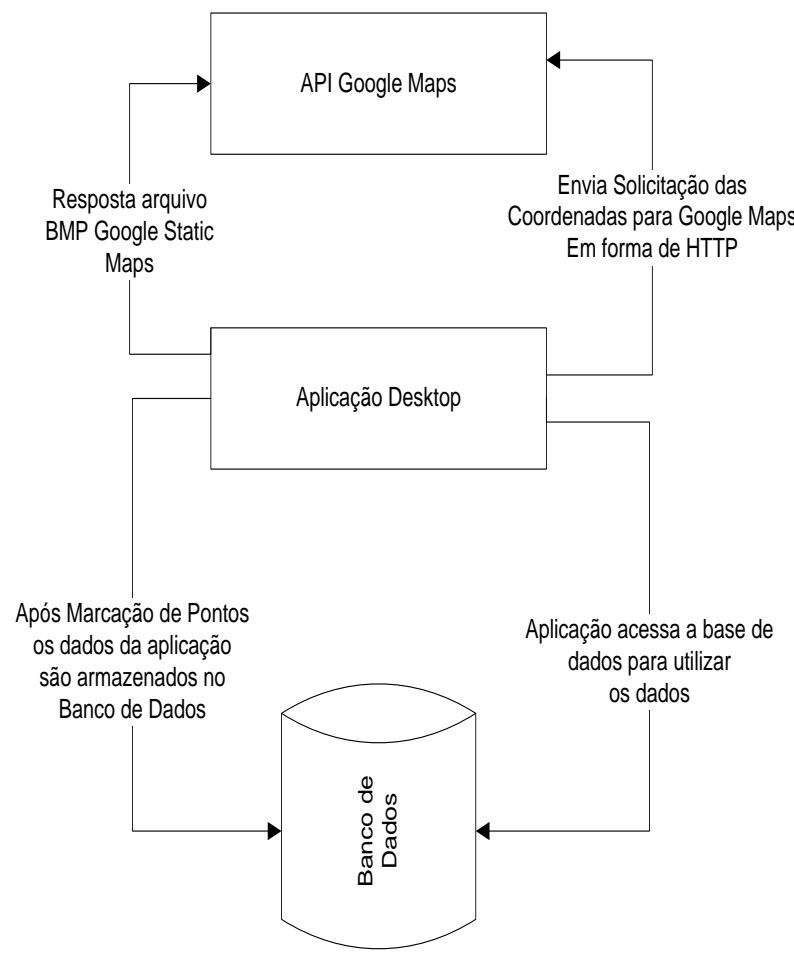

Figura 8. Esquema da funcionalidade desktop acessando os dados do Google Places.

\section{TRABALHO DESENVOLVIDO}

Este trabalho foi idealizado, primeiramente, para catalogar árvores de uma cidade de forma a se ter um levantamento de todas as árvores existentes em determinada região de um município e poder realizar um controle eficaz, dificultando o corte ilegal, que é um problema grave na maioria dos municípios brasileiros, devido ao aquecimento global e problemas com arborização.

Com a utilização da ferramenta se tem a possibilidade de visualizar todas as árvores de determinada avenida de um município ou de determinado bairro.

Depois foram adicionados outros recursos na aplicação que possibilitou a marcação de qualquer outro objeto que fosse desejado, por exemplo: postes, placas de trânsito, semáforos, obstáculos entre outros. Com essa possibilidade de marcar outros objetos a ferramenta deixou de ser útil apenas para secretarias de meio ambiente como também secretarias de trânsito, bem como empresas de fornecimento de energia. Para as empresas de energia a aplicação é útil, a fim de realizar levantamentos das distâncias de postes. Já as secretarias de trânsito podem visualizar posição de placas de trânsito para avaliar se a quantidade das mesmas está adequada para determina via, semáforos para verificar se têm em quantidade suficiente, ou, se é necessário aumentar a quantidade em uma via, entre tantos outros levantamentos que podem vir a ser realizados.

As posições dos objetos no mapa foram calculadas utilizando apenas as latitudes e longitudes dos objetos a serem descritos, estes dados foram obtidos 
diretamente de um documento de texto que é criado no momento em que as imagens são capturadas com a Unidade Móvel de Mapeamento. Esse documento contém dados como posicionamento (latitude, longitude, altitude) e atitude (ângulos do sistema inercial ômega, Phi e Kappa), tanto para as imagens obtidas com a câmara direita, quanto para as imagens obtidas com a câmara da esquerda. O objetivo de se ter duas câmaras é para a possibilidade posterior de se calcular a coordenada do objeto que aparece nas duas imagens (nas duas câmaras) por meio de fotogrametria, que não foi abordado neste trabalho, mas que já foi preparado para tal atividade.

Por meio da aplicação desenvolvida neste trabalho, marca-se nas imagens o objeto desejado e, a partir disso obtêm-se as coordenadas geográficas para aqueles objetos. Ao final, os objetos marcados podem ser visualizados em um mapa. Na Figura 9 é apresentada a modelagem de dados usada neste trabalho.

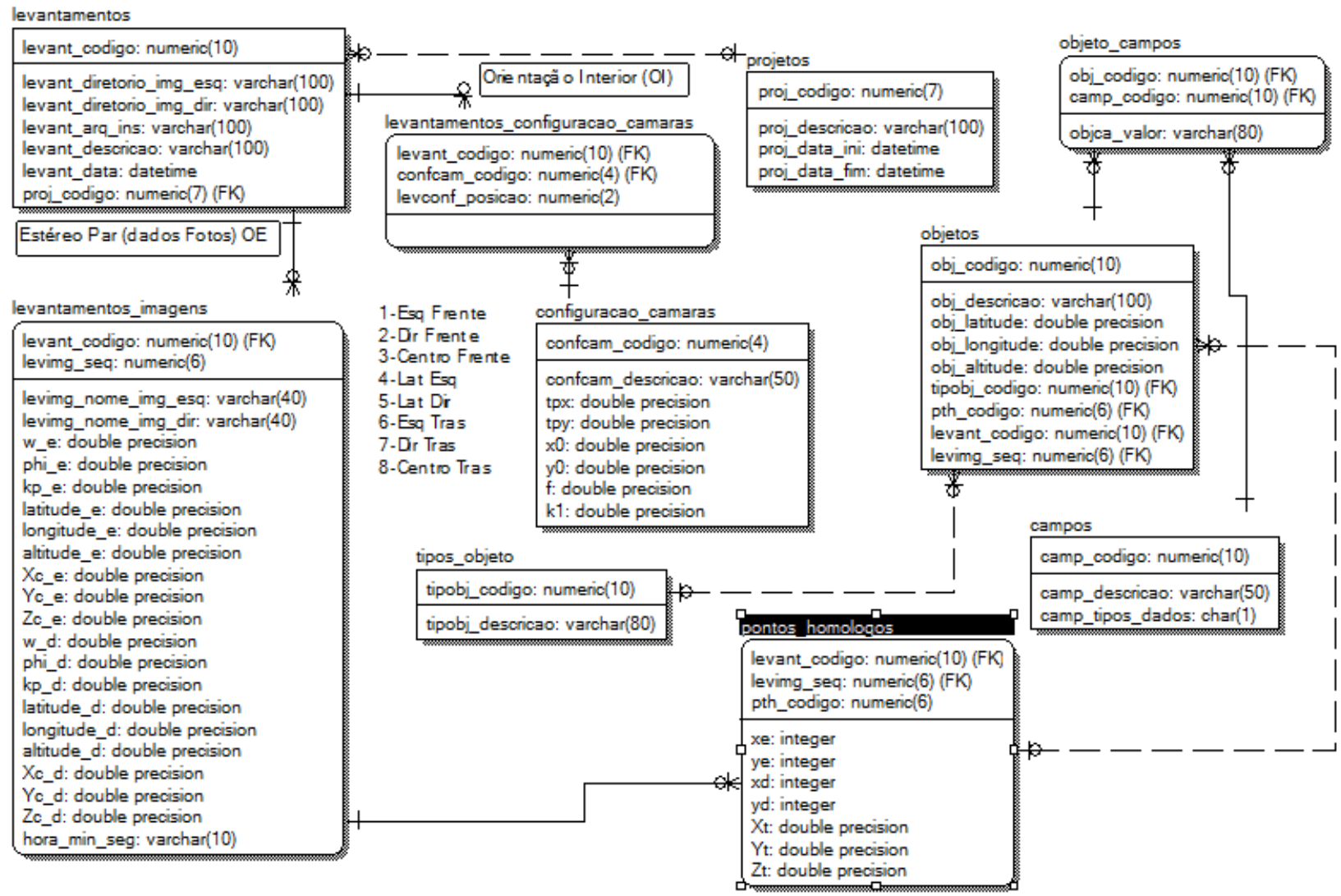

Figura 9. Modelagem de dados da aplicação.

A modelagem possui as seguintes tabelas: "levantamentos", "levantamentos_imagens", "configuracao_camaras",

"levantamento_configuracao_camaras", "projetos", "objetos", "campos", 
"tipos_objeto", "pontos_homologos", "objeto_campos".

Na tabela "projetos" são gravados dados referentes a um determinado projeto. A tabela "configuracao_camaras" é utilizada para salvar os dados referentes as configurações que foram utilizadas nas câmaras.

A tabela “levantamentos_configuracao_camaras" é utilizada para criar um novo levantamento, ela é utilizada para relacionar alguma configuração de câmara para determinado levantamento.

A tabela "levantamentos" é utilizada para guardar os dados de um determinado levantamento.

A tabela "levantamentos_imagens" guarda os dados do arquivo gerado pelo GPS que contém as latitudes, longitudes, altitudes e os ângulos do sistema inercial ômega, phi e kappa dos pares de imagens.

A tabela "tipo_objeto" armazena dados referentes ao tipo de um objeto, por exemplo, se é árvore, poste, lixeira e etc.

A tabela "objetos" é responsável por guardar dados referentes aos objetos, nela são armazenadas as latitudes, longitudes e altitudes, pois é a partir desta tabela que é gerada a camada do mapa.

A tabela "pontos_homologos" é a tabela onde são armazenados os pontos que são marcados nas imagens, que são chamados de pontos homólogos.

Por fim as tabelas "campos" e "objeto_campos" são utilizadas para armazenar os dados que são responsáveis por classificar os objetos. Um exemplo de classificação é a espécie da árvore, ou ainda a altura da mesma.

\section{EXPERIMENTOS}

Foram realizados experimentos com pares de imagens cedidas pela empresa Cartovias. A Cartovias é uma empresa de Engenharia Cartográfica voltada para o mapeamento de ruas e rodovias.

Com esses pares de imagens cedidos, foram realizados experimentos de catalogação de objetos utilizando a ferramenta desenvolvida.

Nos pares de imagens foram catalogados objetos como árvores, postes, placas de trânsito, obstáculos entre outros objetos de uma determinada região da cidade de Presidente Prudente para que fosse realizado o arquivamento e localização destes objetos.

A cada par de fotos foram realizadas as marcações de dois pontos, um em cada foto, e a partir deles foram realizados levantamentos da localização dos objetos na imagem para que fosse gerada a camada do mapa do Google Places, mostrando assim um mapa com a localização desses objetos, 
visando ter um maior controle sobre as

mostra a ferramenta de coleta de pontos da informações dos mesmos. A Figura 10 aplicação desenvolvida neste trabalho.

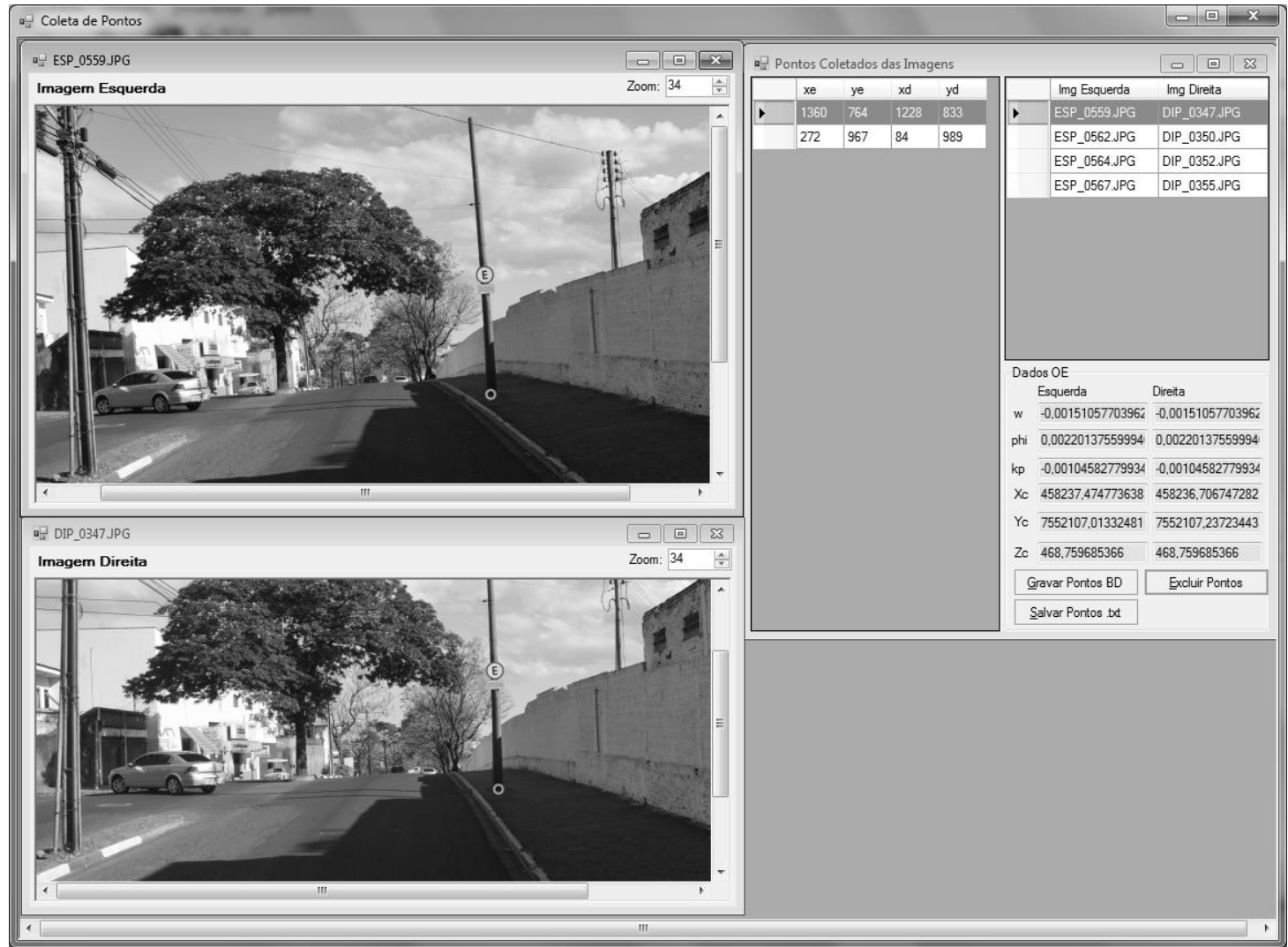

Figura 10. Coleta de pontos da ferramenta.

\section{RESULTADOS}

Os resultados da aplicação (marcação dos pontos nas duas imagens e obtenção das coordenadas geográficas dos objetos desejados) podem ser visualizados por meio de navegadores Web.

Cada marcador na imagem significa um ponto que fora marcado na aplicação e que representa as coordenadas de latitude e longitude do objeto.

Cada um dos objetos na aplicação possui características para a obtenção de resultados precisos, caso haja a necessidade da criação de relatórios na aplicação. Estas características variam entre, espécies de árvores, bem como altura e largura. No caso das lixeiras esses dados podem variar entre altura e largura, até se as mesmas são de coleta seletiva ou não.

Na Figura 11 é mostrado um mapa gerado resultante da aplicação no mapa.

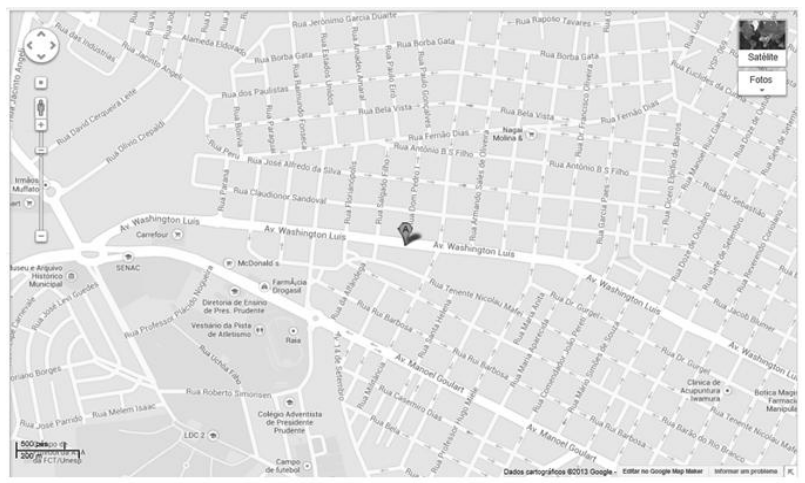

Figura 11. Mapa gerado com a camada gerada pela aplicação. 


\section{CONCLUSÃO}

A proposta deste trabalho foi 0 desenvolvimento de uma aplicação com imagens de dados providos por meio de levantamentos cartográficos e também resultantes de aplicações que tratam esses dados.

A partir do sistema proposto, dados e imagens como os do exemplo apresentado, podem ser buscados, consultados e visualizados em um mapa com suas posições, podendo assim ter um levantamento de determinado objeto em uma cidade.

\subsection{TRABALHOS FUTUROS}

Como trabalho futuro pode ser realizado o cálculo das coordenadas dos objetos que serão exibidos na camada do Google Places, utilizando fototriangulação dos pontos.

\section{REFERÊNCIAS}

CIÊNCIA viva. Disponível em: http://www.cienciaviva.pt/latlong/anterior/g ps.asp. Acesso em: 25 out 2013.

CRAMER, M.; STALLMANN, D. On the use of gps/incertial exterior orientation parameters in airborne photogrammetry. In: FRITSCH; SPILLER (Ed.). Photogrammetric week. Heidelberg, Germany: Wichmann Verlag, 2001.
GUIA do desenvolvedor da api do Google Static Maps. Disponível em: https://developers.google.com/maps/docum entation/staticmaps/index?hl=pt-BR. Acesso em: 10 out 2013.

KALMAN, R. E. A new approach to linear filtering and prediction theory. Journal of Basic Engineering, Transactions ASME Series D, v. 83, p. 35-45, 1960.

MONICO, J. F. G. NAVSTAR-GPS: descrição, fundamentos e aplicações. [S.I.]: Ed. Unesp, 2000.

OLIVEIRA, R. A.; SILVA, J. F. C. Banco de Imagens georreferenciadas no mapeamento terrestre móvel. Boletim de Ciências Geodésicas, Curitiba, v. 7, n. 2, p.3-78, 2001.

SILVA, F. A. Georreferenciamento automático de placas de sinalização com imagens obtidas com um sistema móvel de mapeamento. 2012. 231 p. Tese (Doutorado em Ciências) - Programa de Engenharia Elétrica, Escola de Engenharia de São Carlos, Universidade de São Paulo, São Carlos.

SILVA, J. F. C. et al. Mapeamento de ruas com um Sistema móvel de mapeamento digital. Revista Brasileira de Cartografia, Rio de Janeiro, n. 53, p. 82-91, 2001.

ROQUE, C. G. et al. Georreferenciamento. Revista de Ciências Agro-Ambientais, Alta Floresta, v. 4, n. 1, p. 87-102, 2006.

RUY, R. S. Geração Automática de estereomodelos a partir de imagens digitais georreferenciadas. 2004. 133 f. Dissertação (Mestrado em Ciências Cartográficas) Faculdades de Ciências e Tecnologias, Universidade Estadual Paulista, Presidente Prudente. 